\title{
Ocho documentos barceloneses sobre ventas y cesiones de esclavos (1395)
}

\author{
Josep M. ${ }^{a}$ LLOBET PoRTELLA *
}

\begin{abstract}
RESUMEN ABSTRACT
Se comentan ocho documentos notariales del año 1395 que ofrecen información sobre la venta de tres esclavas y la cesión de un esclavo por un periodo de nueve años a una mujer llamada Violante que era la esposa de

Berenguer de Cortilles, consejero y tesorero de la reina Violante, consorte esta última del monarca catalano-

aragonés Juan I. El precio de las esclavas fue establecido entre los 800 y los 1.050 sueldos, mientras que la cesión del esclavo se valoró en 720 sueldos. Los tres vendedores y el cedente eran de las ciudades de Barcelona y Tarragona.

Eight notarial documents of the year 1395 are commented; these offer information on the sale of three female slaves and the cession of a male slave for a nine year period to a woman called Violante that was the wife of Berenguer de Contilles, consultant and treasurer of the queen Violante, consort this last of the catalanoaragonese king Juan I. The price of the slaves was established between 800 and 1.050 sueldos, while the slave's cession was valued at 720 sueldos. The three salespersons and the grantor were from the cities of Barcelona and Tarragona.
\end{abstract}

Un importante grupo social de la Cataluña medieval era el de los esclavos. Durante el largo período en que se luchó contra los musulmanes o que se mantuvieron guerras exteriores, como las de Cerdeña, la mayor parte de

* UNED. Centro Asociado de Cervera. 
los esclavos eran prisioneros de guerra, puesto que las leyes permitían convertir en esclavo a un enemigo que hubiera sido capturado. Posteriormente, fue el comercio el procedimiento que posibilitó la llegada de nuevos esclavos al territorio catalán. La ciudad de Barcelona, con un buen puerto y un comercio muy activo, ocupó un lugar relevante en la recepción de esclavos ${ }^{1}$.

En los siglos XIV y $\mathrm{XV}$, la mayor parte de los esclavos se encontraban en núcleos urbanos, especialmente en las grandes ciudades. Muy pocos de ellos vivían en el campo. Las mujeres eran empleadas, generalmente, como criadas domésticas, mientras que los hombres eran destinados, habitualmente, a la realización de los trabajos más duros relacionados con la actividad laboral ejercida por su dueño. Sin embargo, algunos de ellos, en la práctica, vivían en una situación similar al resto de los trabajadores asalariados. Aprendían un oficio e, incluso, tenían la posibilidad de poderse comprar su libertad. En otros casos, los esclavos eran manumitidos graciosamente, en atención - según se dice en los documentos- a su fidelidad y buen comportamiento y al hecho de haberse convertido al cristianismo, circunstancia, esta última, que se daba en muchos de ellos, puesto que ello les permitía una mayor aceptación e integración en aquella sociedad medieval profundamente religiosa ${ }^{2}$.

La diferencia más notable que había entre los esclavos y el resto de la población era de naturaleza jurídica. Legalmente privados de libertad y considerados como un bien mueble, podían ser vendidos, comprados o alquilados. A la muerte de su amo, eran incluidos en los inventarios donde se anotaban los bienes del difunto. En la práctica, sin embargo, no era excepcional que se establecieran vínculos afectivos entre los miembros de la familia del amo y el esclavo, especialmente si todos vivían en la misma casa. En algunos testamentos, se establece que a la muerte del propietario del esclavo o poco tiempo después éste quede libre.

Son numerosos los clocumentos que hemos localizado en los archivos que nos pueden facilitar información sobre los esclavos que vivieron en

Uno de los primeros estudios sobre el tema fue el artículo de MIRET I SANS, J., "La esclavitud en Cataluña en los últimos tiempos de la Edad Media", Révue Hispanique, XLI (1917), pp. 2930. Por lo que se refiere a Barcelona, le han seguido, entre otros, SANCHO I MARTínez, D., "La esclavitud en Barcelona en los umbrales de la Edad Moderna", Estudios Históricos y Documentos de los Archivos de Protocolos, VII (1979), pp. 193-270, y SaLICRú I LLUCH, R., «Esclaus i propietaris d'esclaus a la Barcelona de 1424-1425. Una aproximació des del punt de vista sòcio-professional», III Congrés d'història de Barcelona, vol. I, Barcelona, Ajuntament, 1993, pp. 225-232.

2 Una precisa y clara descripción de este colectivo, situado en la Cataluña bajomedieval, se halla en el apartado titulado "Els esclaus» del tomo III de la Història de Catalunya (Dir. Pierre VILAR) que lleva por título «L'expansió baixmedieval (segles XIII-XV)", escrito por Carme BATLLE, pp. 440-445. 
Cataluña durante los siglos XIV y XV. Puesto que la mayor parte de ellos son instrumentos notariales mediante los cuales se legalizaba una compra-venta o una cesión, no resulta difícil obtener los datos personales del vendedor, el comprador y el esclavo, así como el valor de éste y otros datos de interés que nos permiten conocer mejor aquella sociedad medieval. Entre estos textos localizados, hemos elegido ocho documentos que se refieren a la venta de tres esclavas y a la cesión de un esclavo. El comprador o adquirente en todos los casos fue la misma persona, una mujer llamada Violante que era la esposa de Berenguer de Cortilles, consejero y tesorero de la reina Violante, consorte esta última del monarca catalano-aragonés Juan I.

Berenguer de Cortilles era un mercader ciudadano de Zaragoza que acumuló una considerable fortuna con el ejercicio de actividades relacionadas con la administración pública. Durante el reinado de Juan I, arrendó casi todas las cecas que fabricaban moneda real y, en más de una ocasión, compró el derecho a recaudar la décima papal. Por otra parte, en el año 1393, intervino en la obtención de una enorme suma de dinero necesaria para financiar una expedición militar a la isla de Cerdeña. Fue uno de los acusados de mala administración del patrimonio real en el proceso que se siguió a la muerte de Juan I.

Según los documentos estudiados, las esclavas vendidas fueron las siguientes: Caterina, una circasiana de 25 años que se valoró en 880 sueldos 3; Carestina, una tártara también de 25 años que alcanzó la suma de 1.050 sueldos, y Marta, de la cual sólo sabemos que tenía 18 años y valió 800 sueldos. Por otra parte, Antoni, un sarraceno de 25 años, fue cedido durante nueve años por 720 sueldos. Estas cifras parecen indicar que el precio de los varones era muy superior al de las mujeres.

Los vendedores fueron, respectivamente, Manuel de Rajadell, un doncel de Barcelona; Berenguer Gibert, un mercader también de Barcelona, y Joan Ebrí, que era licenciado en leyes y vivía en Tarragona. Jaume de Noguers, otro mercader de Barcelona, era el propietario del esclavo cedido.

A continuación, ofrecemos, transcritos, los ocho documentos notariales que hemos comentado. En cuatro de ellos se legalizaban las transacciones efectuadas y en los otros cuatro los tres vendedores y el cedente reconocian haber recibido el importe de cada venta o cesión. Dichos documentos pertenecen al Fondo Notarial (FN) del Arxiu Històric Comarcal de Cervera $(\mathrm{AHCC})$.

344 libras equivalían a 880 sueldos. El sistema monetario catalán era el siguiente: 1 libra = 20 sueldos $=240$ dineros. 


\section{TEXTOS DOCUMENTALES}

1395, enero, 28. Barcelona

Manuel de Rajadell, doncel, habitante de Barcelona, vende a Violante, esposa de Berenguer de Cortilles, consejero y tesorero de la reina, ciudadano de Zaragoza, una esclava llamada Caterina.

AHCC, FN, Barcelona, 1, Jaume de Corts, Llibre, 1394-1396, f. 203.

Noverint universi quod ego Emanuel de Rayadell, domicellus, habitator civitatis Barchinone, vendo vobis venerabili Violanti, uxori venerabilis Berengarii de Cortilles, illustrissime domine regine consiliarii et thesaurarii civisque Cesarauguste, presenti, et vestris quandam sclavam, servam et captivam meam, de genere xarcorum, vocatam Cathalina, etatis viginti quinque annorum vel circa. Hanc autem venditionem facio vobis et vestris et quibus velitis de predicta serva et captiva sicut melius dici potest et intelligi ad salvamentum et bonum intellectum vestri et vestrorum. Inducens vos in corporalem possessionem dicte serve per traditionem, quam vobis facio de eadem. Preterea, ex causa huiusmodi venditionis predicte, cedo et mando vobis omnia iura omnesque actiones reales et personales, mixtas, utiles et directas, ordinarias et extraordinarias et alias quascunque michi competentes et competere debentes et valentes in predicta serva et contra quascunque personas ratione eiusdem. Quibus iuribus et actionibus supra dictis possitis vos et vestri et quos volueritis uti, agere et experiri et omnia alia facere in iudicio et extra iudicium quecunque et quem admodum ego facere poteram ante presentem venditionem et iurium cessionem et possem nunc et etiam postea quandocunque. Ego enim ffacio et constituo vos in hiis dominam et procuratricem ut in rem vestram propriam ad faciendum inde vestre libitum voluntatis. Pro pretio vero predictorum que vobis vendo dedistis et solvistis michi et confiteor me a vobis habuisse et recepisse quadraginta quatuor libras barchinonenses de terno. Et ideo, renuntiando exceptioni peccunie non numerate et non solute et doli mali et actioni in factum et legi qua deceptis ultra dimidiam iusti pretii subvenitur et omni alii iuri, rationi et consuetudini contra hec repugnantibus, do et ex certa scientia remitto vobis donatione irrevocabili inter vivos si quid predicta serva quam vobis vendo plus modo valet vel de cetero valebit pretio supra dicto. Insuper convenio et promitto vobis quod predictam Catalinam pro serva et captiva vestra faciam vos et vestros et quos volueritis perpetuo habere, tenere et possidere in pace contra omnes personas. Et quod tenebor vobis et vestris semper de vitio et evictione et de morbo caduco et 
absconso vitio ipsius serve et captive ad usum et consuetudinem Barchinone, necnon de deffensione eiusdem ac de refectione et restitutione omnium expensarum ac interesse vestri et vestrorum in iudicio et extra iudicium omni exceptione remota. Pro quibus omnibus et singulis complendis et firmiter attendendis, obligo vobis et vestris omnia bona mea, mobilia et inmobilia, habita et habenda. Premissa igitur omnia et singula facio, paciscor et promitto ego dictus Emanuel vobis dicte venerabili Violanti et vestris et notario infra scripto tanquam publice persone pro vobis et pro aliis etiam personis omnibus quarum interest et intererit recipienti et paciscenti ac etiam legittime stipulanti. Actum est hoc Barchinone XXVIII. ${ }^{a}$ die ianuarii anno a nativitate Domini $\mathrm{M}^{\circ} \mathrm{CCC} .{ }^{\circ} \mathrm{XC} .{ }^{\circ}$ quinto. Sig+num Emanuelis de Rayadell predicti, qui hec laudo et firmo.

Testes huius rei sunt venerabilis lohannes Çafont, de thesauraria, civis Barchinone, et Bernardus dez Canall, notarius de officio magistri rationalis curie domini regis.

1395, febrero, 17. Barcelona

Manuel de Rajadell, doncel, habitante de Barcelona, reconoce haber recibido de Violante, esposa de Berenguer de Cortilles, consejero y tesorero de la reina, 44 libras, que son el precio de una esclava llamada Caterina.

AHCC, FN, Barcelona, 1, Jaume de Corts, Llibre, 1394-1396, f. 203 v.

Sit omnibus notum quod ego Emanuel de Rayadell, domicellus, habitator civitatis Barchinone, confiteor et recognosco vobis venerabili Violanti, uxori venerabilis Berengarii de Cortilles, illustrissime domine regine consiliarii et thesaurarii, et vestris quod tradidistis et solvistis michi ad meam voluntatem omnes illas quadraginta quatuor libras barchinonenses de terno, pro quibus seu quarum pretio ego vendidi vobis et quibus velitis quandam sclavam, servam et captivam meam, Cathalina nuncupatam, de genere xarcorum, prout de ipsa venditione plene constat instrumento publico inde facto in posse notarii infra scripti XXVIII. ${ }^{a}$ die ianuarii anno infra scripto. Et ideo, renuntiando exceptioni peccunie non numerate et non solute et doli mali et actioni in factum ac omni alii iuri, rationi et consuetudini contra hec repugnantibus, facio vobis de predictis quadraginta quatuor libris bonum et perpetuum finem et pactum et presentem apocam de soluto in posse notarii infra scripti vallatum stipulatione solemni. Actum est hoc Barchinone XVII. ${ }^{a}$ die februarii anno a nativitate Domini $\mathrm{M} .{ }^{\circ} \mathrm{CCC} .{ }^{\circ} \mathrm{XC} .{ }^{\circ}$ quinto. Sig+num Emanuelis predicti, qui hec concedo et firmo. 
Testes huius rei sunt Guillemus Ferrarii, notarius de thesauraria domini regis, et venerabilis Petrus Raymundi de Coponibus, domicellus, dominus castri de Durban, vicarie Cervarie.

1395, mayo, 26. Barcelona

Berenguer Gibert, mercader, ciudadano de Barcelona, vende a Violante, esposa de Berenguer de Cortilles, ciudadano de Zaragoza, una esclava Ilamada Carestina.

AHCC, FN, Barcelona, 1, Jaume de Corts, Llibre, 1394-1396, f. 39.

In civitate Barchinone $\mathrm{XXVI.}{ }^{a}$ die madii anno a nativitate Domini $\mathrm{M}^{\circ}$ CCC. ${ }^{\circ} \mathrm{XC} .^{\circ}$ quinto.

Noverint universi quod ego Berengarius Gibert, mercator, civis Barchinone, gratis et ex certa scientia vendo et titulo venditionis concedo vobis venerabili domine Violanti, uxori honorabilis Berengarii de Cortilles, civis Cesarauguste, presenti et acceptanti, et vestris et quibus velitis quandam sclavam, servam et captivam meam, vocatam Carestina, de genere tartarorum, in etate viginti quinque annorum vel inde circa, plenaria constituta. Hanc autem venditionem et titulo venditionis concessionem facio dicto nomine vobis et vestris et quibus volueritis de predicta sclava sicut melius dici potest et intelligi ad vestrum vestrorumque salvamentum et bonum etiam intellectum. Inducens vos in possessionem corporalem de predicta sclava per traditionem, quam vobis facio de eadem. Et, ex causa huiusmodi venditionis, do, cedo, transfero atque mando vobis et vestris et quibus velitis omnia iura omnesque voces et actiones reales et personales, mixtas, utiles et directas, ordinarias et extraordinarias et alias quascunque que michi competunt et competere possunt ac debent insuper predicta sclava quam vobis vendo et contra quascunque personas et res et in quibuscunque bonis ratione et occasione eiusdem. Quibus iuribus et actionibus supra dictis possitis vos et vestri et quos volueritis uti et experiri, agendo scilicet et respondendo, deffendendo, excipiendo, proponendo et replicando et omnia alia et singula faciendo et libere exercendo in iudicio et extra iudicium quecunque et quem admodum ego facere possem nunc et etiam postea quandocunque. Ego enim facio et constituo vos et vestros in hiis dominos et procuratores ut in rem vestram propriam ad faciendum inde vestre libitum voluntatis sine contradictione et impedimento mei et meorum et alterius cuiuscunque persone. Pro pretio vero predicte sclave quam vobis vendo dedistis et solvistis michi et confiteor me a vobis habuisse et recepisse mille et quinquaginta solidos barchinonenses de terno. Et ideo, 
renuntiando exceptioni peccunie non numerate et pretii predicti non habiti et non recepti et legi que subvenit deceptis ultra dimidiam iusti pretii et exceptioni de dolo malo et in factum actioni et omni alii iuri, rationi et consuetudini contra hec repugnantibus, do et remitto vobis et vestris si quid predicta sclava quam vobis vendo plus modo valet aut a modo valere poterit pretio ante dicto. Insuper convenio et promito vobis quod predictam sclavam quam vobis vendo, non furto habitam nec de pallia ac treuga domini regis sed de bona guerra, faciam vos et vestros et quos volueritis habere, tenere et pacifice possidere in pace contra cunctas personas. Et quod tenebor vobis et vestris de firma et legali evictione eiusdem et de restitutione omnium missionum, expensarum, damnorum et interesse, litis et extra, et de morbo absconso atque caduco et aliis vitiis absconsis iuxta iuris dispositionem et etiam alias ad usum et consuetudinem Barchinone. Et, pro hiis complendis et firmiter attendendis, tenendis et observandis, obligo vobis et vestris omnia bona mea, mobilia et inmobilia, ubique habita et habenda. Et etiam iuro per dominum Deum et eius sancta IIII. or evangelia manibus meis corporaliter tacta predicta omnia et singula attendere et complere, tenere et observare, et in nullo contra facere vel venire iure aliquo, causa vel etiam ratione. Hec igitur omnia et singula supra dicta facio, paciscor, convenio et promito ego dictus Berengarius Gibert vobis dicte venerabili domine Violanti et vestris et notario etiam infra scripto tanquam publice persone pro vobis et pro personis omnibus quarum interest et intererit recipienti et paciscenti ac etiam legittime stipulanti. Actum est hoc Barchinone XXVI. ${ }^{a}$ die madii anno a nativitate Domini $\mathrm{M} .{ }^{\circ} \mathrm{CCC} .{ }^{\circ} \mathrm{XC} .{ }^{\circ}$ quinto. Sig+num Berengarii Gibert predicti, qui hec laudo, concedo, firmo et iuro.

Testes huius rei sunt venerabiles Guillemus de Ferreres, Guillemus Cardona, de thesauraria domine regine cives, et Guillemus Coral, curritor auralium civitatis Barchinone.

1395, julio, 9. Barcelona

Berenguer Gibert, mercader, ciudadano de Barcelona, reconoce haber recibido de Violante, esposa de Berenguer de Cortilles, ciudadano de Zaragoza, mil cincuenta sueldos, que son el precio de una esclava llamada Carestina.

AHCC, FN, Barcelona, 1, Jaume de Corts, Llibre, 1394-1396, f. 39 v.

Civitate Barchinone IX. ${ }^{\text {a }}$ die iulii anno predicto.

Sit omnibus notum quod ego Berengarius Gibert, mercator, civis Barchinone, confiteor et recognosco vobis venerabili domine Violanti, uxori 
honorabilis Berengarii de Cortilles, civis Cesarauguste, et vestris quod dedistis et exsolvistis michi recipienti numerando bene et plenarie voluntati mee omnes illos mille et quinquaginta solidos barchinonenses de terno, quibus seu quorum pretio vendidi vobis et vestris et quibus velitis quandam sclavam, servam et captivam meam, vocatam Carestina, de genere tartarorum, in etate viginti quinque annorum vel inde circa, plenariam constitutam, prout de ipsa venditione plene constat per instrumentum inde factum in posse notarii infra scripti XXVI. ${ }^{a}$ die madii anno subscripto. Et ideo, renuntiando exceptioni peccunie non numerate et non solute et doli, facio vobis in posse notarii subscripti de predictis mille quinquaginta solidis barchinonensibus michi per vos exsolutis bonum et perpetuum finem et pactum de ulterius non petendo et non agendo per solemnem stipulationem vallatum ac presens apoche instrumentum in testimonium premissorum. Actum est hoc Barchinone nona die iulii anno a nativitate Domini $\mathrm{M}{ }^{\circ} \mathrm{CCC}^{\circ} \mathrm{XC} .^{\circ}$ quinto. Sig+num Berengarii Gibert predicti, qui hec concedo et firmo.

Testes huius rei sunt Ffranciscus Vinyoles, ville Cardone, et Guillemus Cardona, de thesauraria domini regis.

1395, junio, 5. Barcelona

Joan Ebri, licenciado en leyes, ciudadano de Tarragona, vende a Violante, esposa de Berenguer de Cortelles, consejero y tesorero de la reina, ciudadano de Zaragoza, una esclava llamada Marta.

AHCC, FN, Barcelona, 1, Jaume de Corts, Llibre, 1394-1396, f. 199.

In civitate Barchinone quinta die iunii anno a nativitate Domini $\mathrm{M}^{\circ}{ }^{\circ}$ $\mathrm{CCC}^{\circ} \mathrm{XC} .{ }^{\circ} \mathrm{V} \cdot{ }^{\circ}$.

Noverint univesi quod ego lohannes Ebrí, in legibus licentiatus, civis Terracone, vendo vobis venerabili Violanti, uxori venerabilis Berengarii de Cortelles, illustrissime domine regine Aragonum consiliarii et thesaurarii civisque Cesaraugiste, et vestris quandam sclavam, servam et captivam meam, vocatam Marta, etatis XVIII. ${ }^{\circ}$ annorum vel circa. Hanc autem venditionem facio vobis et vestris et quibus velitis de predicta serva et captiva sicut melius dici potest et intelligi ad salvamentum et bonum intellectum vestri et vestrorum. Inducens vos in corporalem possessionem dicte serve per traditionem, quam vobis facio de eadem. Preterea, ex causa huiusmodi venditionis predicte, cedo et mando vobis omnia iura omnesque actiones reales et personales, mixtas, utiles et directas, ordinarias et extraordinarias et alias quascunque michi competentes et competere debentes et valentes in pre- 
dicta serva et contra quascunque personas ratione eiusdem. Quibus iuribus et actionibus supra dictis possitis vos et vestri et quos volueritis uti, agere et experiri et omnia alia facere in iudicio et extra iudicium quecunque et quem admodum ego facere poteram ante presentem venditionem et iurium cessionem et possem nunc et etiam postea quandocunque. Ego enim facio et constituo vos dominam et procuratricem ut in rem vestram propriam ad faciendum inde vestre libitum voluntatis. Pro pretio vero predictorum que vobis vendo dedistis et solvistis michi et confiteor me a vobis habuisse et recepisse octingentos solidos barchinonenses de terno. Et ideo, renuntiando exceptioni peccunie non numerate et no solute et doli mali et actioni in factum et legi qua deceptis ultra dimidiam iusti pretii subvenitur et omni alii iuri, rationi et consuetudini contra hec repugnantibus, do et ex certa scientia remito vobis donatione irrevocabili inter vivos si quid predicta serva quam vobis vendo plus modo valet vel de cetero valebit pretio supra dicto. Insuper convenio et promitto vobis quod predictam Martam pro serva et captiva vestra faciam vos et vestros et quos volueritis perpetuo habere, tenere et possidere in pace contra omnes personas. Et quod tenebor vobis et vestris semper de vitio et evictione et de morbo caduco et absconso vitio ipsius serve et captive ad usum et consuetudinem Barchinone, necnon de defensione eiusdem ac de refectione et restitutione omnium expensarum ac interesse vestri et vestrorum in iudicio et extra iudicium omni exceptione remota. Pro quibus omnibus et singulis complendis et firmiter attendendis, obligo vobis et vestris omnia bona mea, mobilia et inmobilia, habita et habenda. Premissa igitur omnia et singula facio, paciscor et promito ego dictus lohannes Ebrí vobis dicte venerabili Violanti et vestris et notario infra scripto tanquam publice persone pro vobis et pro aliis etiam personis omnibus quarum interest et intererit recipienti et paciscenti ac etiam legittime stipulanti. Actum est hoc Barchinone quinta die iunii anno a nativitate Domini $\mathrm{M} .{ }^{\circ} \mathrm{CCC} .{ }^{\circ} \mathrm{XC} .{ }^{\circ}$ quinto. Sig+num lohannis Ebri, venditoris, predicti, qui hec laudo, concedo et firmo.

Testes huius rei sunt venerabilis Guillemus de Farreres, procurator reginalis, civitatis et campi Terracone, et lohannes Calvet, habitator eiusdem civitatis.

1395, junio, 5. Barcelona.

Joan Ebri, licenciado en leyes, ciudadano de Tarragona, reconoce haber recibido de Violante, esposa de Berenguer de Cortelles, consejero y tesorero de la reina, ciudadano de Zaragoza, ochocientos sueldos, que son el precio de una esclava llamada Marta.

AHCC, FN, Barcelona, 1, Jaume de Corts, Llibre, 1394-1396, f. 199 v. 
Sit omnibus notum quod ego lohannes Ebrí, in legibus licentiatus, civis Terracone, confiteor et recognosco vobis venerabili Violanti, uxori venerabilis Berengarii de Cotilles, illustrissime domine regine Aragonum consiliarii et thesaurarii civisque Cesarauguste, et vestris quod tradidistis et solvistis michi ad meam voluntatem omnes illos octingentos solidos monete barchinonensis de terno, pro quibus seu quorum pretio ego vendidi vobis et quibus velitis quandam sclavam, servam et captivam meam, Marta nuncupatam, ut de ipsa venditione plene constat instrumento publico inde facto in posse notarii infra scripti die et anno subscriptis. Et ideo, renuntiando exceptioni peccunie non numerate et non solute et doli mali et actioni in factum ac omni alii iuri, rationi et consuetudini contra hec repugnantibus, facio vobis de predictis octingentis solidis bonum et perpetuum finem et pactum et presentem apocam de soluto in posse notarii infra scripti vallatum stipulatione solemni. Actum est hoc Barchinone quinta die iunii anno a nativitate Domini M. ${ }^{\circ} \mathrm{CCC}{ }^{\circ} \mathrm{XC} .^{\circ}$ quinto. Sig+num lohannis Ebrí predicti, qui hec concedo et firmo.

Testes huius rei sunt venerabilis Guillemus de Farreres, procurator reginalis, civitatis et campi Terracone, et lohannes Calvet, habitator eiusdem civitatis.

1395, diciembre, 20. Barcelona

Jaume de Noguers, mercader, ciudadano de Barcelona, cede durante nueve años a Violante, esposa de Berenguer de Cortilles, ciudadano de Zaragoza, un esclavo llamado Antoni.

AHCC, FN, Barcelona, 1, Jaume de Corts, Llibre, 1394-1396, f. 38.

In civitate Barchinone $X X{ }^{a}$ die decembris anno a nativitate Domini $\mathrm{M}^{\circ}$ CCC. ${ }^{\circ} \mathrm{XC} .{ }^{\circ}$ quinto.

Noverint universi quod ego lacobus dez Noguers, mercator, civis Barchinone, gratis et ex certa scientia vendo et titulo venditionis concedo vobis venerabili domine Violanti, uxori venerabilis Berengarii de Cortelles, civis Cesarauguste, presenti et acceptanti, et vestris et quibus velitis, de proximo instanti festo Sancti Michaelis mensis septembris ad novem annos tunc inmediate sequentes et continue numerandos et completos et hinc ad dictum festum, quendam sclavum, servum et captivum meum, per dictum tempus, vocatum Anthonius, de genere sarracenorum, in etate viginti quinque annorum vel inde circa, plenarius constitutus. Hanc autem venditionem et titulo venditionis concessionem facio vobis et vestris et quibus 
volueritis de predicto servo sive, ad dictum tempus, sclau sicut melius dici potest et intelligi ad vestrum vestrorumque salvamentum et bonum etiam intellectum. Inducens vos in possessionem corporalem de predicto servo per traditionem, quam vobis facio de eodem. Et, ex causa huiusmodi venditionis, do, cedo, transfero atque mando vobis et vestris et quibus velitis omnia iura omnesque voces et actiones reales et personales, mixtas, utiles et directas, ordinarias et extraordinarias et alias quascunque que michi competunt et competere possunt ac debent in et super dicto sclavo quem vobis vendo et contra quascunque personas et res et in quibuscunque bonis ratione et occasione eiusdem. Quibus iuribus et actionibus supra dictis possitis vos et vestri et quos volueritis uti et experiri, agendo scilicet et respondendo, deffendendo, excipiendo, proponendo et replicando et omnia alia et singula faciendo et libere exercendo in iudicio et extra iudicium quecunque et quem admodum ego facere possem nunc et etiam postea quandocumque. Ego enim facio et constituo vos et vestros in his dominos et procuratores ut in rem vestram propriam ad faciendum inde vestre libitum voluntatis sine contradictione et impedimento mei et meorum et alterius cuiuscunque persone. Pro pretio vero predicti servi quem vobis vendo dedistis et solvistis michi et confiteor me a vobis habuisse et recepisse septingentos viginti solidos barchinonenses de terno. Et ideo, renuntiando exceptioni peccunie non numerate et pretii predicti non habiti et non recepti et legi que subvenit deceptis ultra dimidiam iusti pretii et exceptioni de dolo malo et in factum actioni et omni alii iuri, rationi et consuetudini contra hec repugnantibus, do et remitto vobis et vestris si quid predictus servus quem vobis vendo plus modo valet aut a modo valere poterit pretio ante dicto. Insuper convenio et promito vobis quod predictum servum quem vobis vendo, non furto habitum nec de pallia ac treuga domini regis sed de bona guerra, faciam vos et vestros et quos volueritis, per totum supra dictum tempus, habere, tenere et pacifice possidere in pace contra cunctas personas. Et quod tenebor vobis et vestris, toto tempore supra dicto, de firma et legali evictione eiusdem et de restitutione omnium missionum, expensarum, damnorum et interesse, littis et extra, et de morbo absconso atque caduco et aliis vitiis absconsis iuxta iuris dispositionem et etiam alias ad usum et consuetudinem Barchinone. Et, pro hiis complendis et firmiter attendendis, tenendis et observandis, obligo vobis et vestris omnia bona mea, mobilia et inmobilia, ubique habita et habenda. Et etiam iuro per dominum Deum et eius sancta quatuor evangelia manibus meis corporaliter tacta predicta omnia et singula attendere et complere, tenere et observare, et in nullo contra facere vel venire iure aliquo, causa vel etiam ratione. Hec igitur omnia et singula supra dicta facio, paciscor, convenio et promito ego dictus lacobus dez Noguers vobis dicte venerabi- 
li domine Violanti et vestris et notario etiam infra scripto tanquam publice persone pro vobis et pro personis omnibus quarum interest et intererit recipienti et paciscenti ac etiam ligittime stipulanti. Actum est hoc Barchinone $\mathrm{XX} .{ }^{\mathrm{a}}$ die decembris anno a nativitate Domini $\mathrm{M} .{ }^{\circ} \mathrm{CCC} .{ }^{\circ} \mathrm{XC} .{ }^{\circ}$ quinto. Sig+num lacobi dez Noguers predicti, qui hec laudo, concedo, firmo et iuro.

Testes huius rei sunt venerabiles Petrus Cardona et Ffranciscus dez Puitg, mercatores, cives Barchinone.

1395, diciembre, 20. Barcelona

Jaume de Noguers, mercader, ciudadano de Barcelona, reconoce haber recibido 720 sueldos de Violante, esposa de Berenguer de Cortilles, ciudadano de Zaragoza, que son el precio de la cesión durante nueve años de un esclavo llamado Antoni.

AHCC, FN, Barcelona, 1, Jaume de Corts, Llibre, 1394-1396, f. 38 v.

Die predicta in dicta civitate.

Sit omnibus notum quod ego lacobus dez Noguers, mercator, civis Barchinone, confiteor et recognosco vobis venerabili domine Violanti, uxori honorabilis Berengarii de Cortilles, civis Cesarauguste, et vestris quod dedistis et exsolvistis michi recipienti numerando bene et plenarie voluntati mee omnes illos septingentos viginti solidos barchinonenses de terno, quibus seu quorum pretio vendidi vobis et vestris et quibus velitis quendam sclavum, servum et captivum meum, vocatum Anthonius, de genere sarracenorum, in etate viginti quinque annorum vel inde circa, plenarius constitutus, ad certum tempus in instrumento venditionis eiusdem servi contentum, prout constat de ipsa venditione per instrumentum publicum inde factum in posse notarii infra scripti $X X{ }^{a}$ die decembris anno subscripto. Et ideo, renuntiando exceptioni peccunie non numerate et non solute et doli, facio vobis in posse notarii subscripti de predictis septingentis viginti solidis barchinonensibus michi per vos exsolutis bonum et perpetuum finem et pactum de ulterius non petendo et non agendo per sollemnem stipulationem vallatum ac presens apoce instrumentum in testimonium premissorum. Actum est hoc Barchinone $X X{ }^{a}$ die decembris anno a nativitate Domini $\mathrm{M} .{ }^{\circ} \mathrm{CCC} .{ }^{\circ} \mathrm{XC}$ quinto. Sig+num lacobi dez Noguers predicti, qui hec concedo et firmo.

Testes huius rei sunt venerabiles Petrus Cardona et Ffranciscus des Puitg, mercatores, cives Barchinone. 